\title{
Low-Thrust Transfer Design of Low-Observable Geostationary Earth Orbit Satellite
}

\author{
Bing Hua ${ }^{1,2}$ and Zhujun Shao ${ }^{2}$ \\ ${ }^{1}$ State Key Laboratory of Virtual Reality Technology and Systems, Beihang University, Beijing 100091, China \\ ${ }^{2}$ College of Astronautics, Nanjing University of Aeronautics \& Astronautics, Nanjing 210016, China \\ Correspondence should be addressed to Bing Hua; bing.hua@nuaa.edu.cn
}

Received 4 August 2015; Revised 10 November 2015; Accepted 24 November 2015

Academic Editor: Paul Williams

Copyright (c) 2015 B. Hua and Z. Shao. This is an open access article distributed under the Creative Commons Attribution License, which permits unrestricted use, distribution, and reproduction in any medium, provided the original work is properly cited.

\begin{abstract}
With radar and surface-to-air missiles posing an increasing threat to on-orbit spacecraft, low-observable satellites play an important role in low-thrust transfers. This paper presents the design for a low-thrust geostationary earth orbit (GEO) transfer control strategy which takes into consideration the low-observable constraint and discusses Earth shadow and perturbation. A control parameter optimization addresses the orbit transfer problem, and five thrust modes are used. Simulation results show that the method outlined in this paper is simple and feasible and results in reduced transfer time with a small amount of calculation. The method therefore offers a useful reference for low-thrust GEO transfer design.
\end{abstract}

\section{Introduction}

Creation of a low-observable satellite is accomplished by lowobservable technology, which makes it difficult or impossible to avoid detection of satellites in orbit by hostile enemy forces [1-6]. Adding a low-observability module to the spacecraft's overall design is key to improving operational effectiveness and satellite survivability.

A geostationary satellite is usually launched from ground to Low Earth Orbit (LEO) or Middle Earth Orbit (MEO) rather than to GEO. Since the main threat to LEO and MEO satellites comes from radar, a great deal of emphasis in lowobservability design is placed on radar analysis. The radar cross section (RCS) is the designers' only controllable factor in radar detection. Contemporary work on low observability has its roots in efforts at reducing the RCS of a spacecraft, falling into two categories: low-observable shape design and flight attitude planning [1-8]. The former is a simple way of reducing the range at which radar can detect the spacecraft; however, contouring the surface of a spacecraft to reduce the RCS equally in all directions is not possible. As a result, the latter is typically supplemented in designs in order to produce better results.
The application of electric propulsion in geostationary orbit platforms is inevitable for the development of the aerospace. Used for spacecraft applications in Earth orbit, such as station-keeping, orbit-raising, and orbit transfer, Boeing-702sp is an all-electric propulsion satellite with a specific impulse of more than 3800 seconds [9-12]. A great deal of research has been directed toward solving the lowthrust transfer problem. Most of that research has been based on optimal control theory. The numerical solution incorporates both direct and indirect methods, due to their high accuracy [13-16]. As is typical with these methods, the solution is often difficult to derive, requiring a complex initial guess and tedious iteration for convergence.

Electric propulsion, low thrust, and highly specific impulses have led to greatly improved fuel efficiency at the expense of relatively long transfer times. For these reasons, satellites can be easily caught by detection systems during low-thrust GEO transfer. Existing research on low-thrust transfer emphasizes optimum techniques, giving little consideration to the satellite attitude constraint of control. This paper models an optimization scheme of the low-thrust transfer based on low-observable technology, which can 


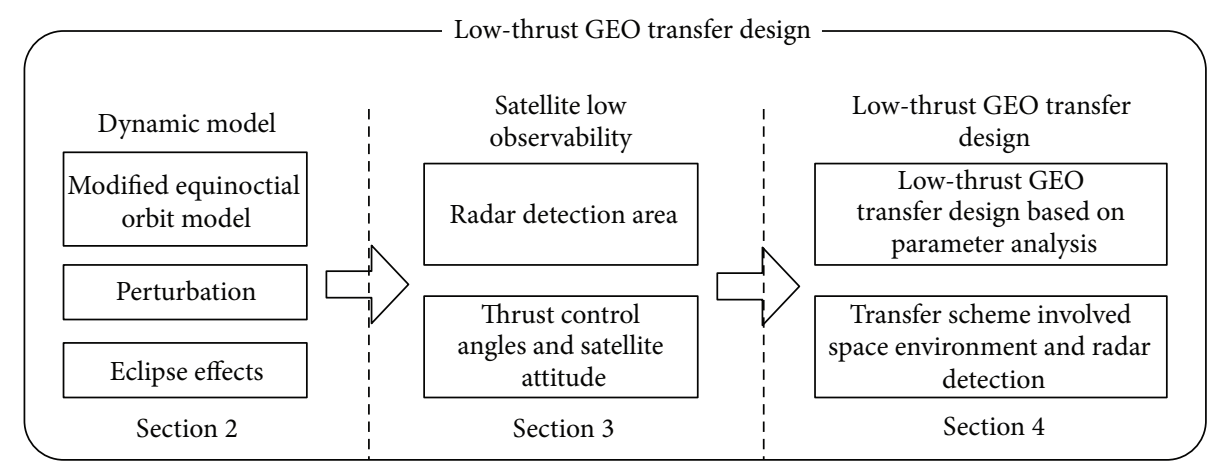

Figure 1: Paper structure.

reduce the visibility of satellites. Furthermore, the optimization includes space environment (Earth shadow and perturbation). Offering the advantages of short time, a small amount of calculation, and no complex initial guess, the method designed in this paper can provide a valuable reference for low-thrust GEO transfer design.

The paper is organized in five distinct sections, as represented in Figure 1. The introduction above has described lowobservable satellites and low-thrust transfer optimization techniques. In Section 2, Earth shadow and perturbation in orbit transfer are analyzed. In Section 3, a mathematical model of low-observable constraint is demonstrated, including the radar detection area and the relationship of thrust control angle and attitude angle. Section 4 presents a method based on control parameter analysis to solve minimum-time transfer. Finally, we summarize the paper.

\section{Dynamic Model}

2.1. Modified Equinoctial Orbit Model. The dynamical equations of motion for a thrusting spacecraft can be established by the classical orbital elements: the semimajor axis $a$, the eccentricity $e$, the inclination $i$, the right ascension $\Omega$, the argument of perigee $\omega$, and the mean anomaly $M$ :

$$
\begin{aligned}
\dot{a}= & \frac{2 a^{2} e \sin v}{h} f_{r}+\frac{2 a^{2} p}{h r} f_{t} \\
\dot{e}= & \frac{1}{h} p \sin v f_{r}+\frac{1}{h}[(p+r) \cos v+r e] f_{t} \\
\dot{i}= & \frac{r \cos (\omega+v)}{h} f_{n} \\
\dot{\Omega}= & \frac{r \sin (\omega+v)}{h \sin i} f_{n} \\
\dot{\omega}= & -\frac{p \cos v}{h e} f_{r}+\frac{(p+r) \sin v}{h e} f_{t} \\
& +\frac{r \sin v(\omega+v) \cos i}{h \sin i} f_{n} \\
\dot{M}= & n+\frac{1}{a e h}\left[(p \cos v-2 e r) f_{r}-(p+r) \sin v f_{t}\right]
\end{aligned}
$$

where $p=a\left(1-e^{2}\right), h=\sqrt{\mu p}, n=\sqrt{\mu / a^{2}}, r=p /(1+e \cos v)$ with Earth's gravitational coefficient $\mu$ and the true anomaly $v$. Performing analyses of transfer orbits using classical orbital elements is a straightforward task, but singularities are exhibited for zero eccentricity and inclinations of $0^{\circ}$ and $90^{\circ}$.

To eliminate these deficiencies, a modified set of equinoctial orbit elements $\left(p, e_{x}, e_{y}, h_{y}, h_{x}\right.$, and $\left.L\right)$ is frequently used. $P$ is the semilatus rectum, $\left(e_{x}, e_{y}\right)$ is the eccentricity vector, $\left(h_{x}, h_{y}\right)$ is the inclination vector, and $L$ is the true longitude. The relationship between the modified equinoctial elements and the classical orbital elements is given by

$$
\begin{aligned}
p & =a\left(1-e^{2}\right) \\
e_{x} & =e \cos (\omega+\Omega) \\
e_{y} & =e \sin (\omega+\Omega) \\
h_{x} & =\tan \left(\frac{i}{2}\right) \cos \Omega \\
h_{y} & =\tan \left(\frac{i}{2}\right) \sin \Omega \\
L & =\omega+\Omega+v .
\end{aligned}
$$

The equations of motion, written in terms of the modified equinoctial elements, are

$$
\begin{aligned}
\dot{p} & =\sqrt{\frac{p}{\mu}} \frac{2 p}{\omega} f_{t} \\
\dot{e}_{x} & =\sqrt{\frac{p}{\mu}}\left\{f_{r} \sin L+\left[(1+\omega) \cos L+e_{x}\right] \frac{f_{t}}{\omega}\right. \\
& \left.-\left(h_{x} \sin L-h_{y} \cos L\right) \frac{e_{y} f_{n}}{\omega}\right\} \\
\dot{e}_{y} & =\sqrt{\frac{p}{\mu}}\left\{-f_{r} \cos L+\left[(1+\omega) \sin L+e_{y}\right] \frac{f_{t}}{\omega}\right. \\
& \left.+\left(h_{x} \sin L-h_{y} \cos L\right) \frac{e_{x} f_{n}}{\omega}\right\}
\end{aligned}
$$




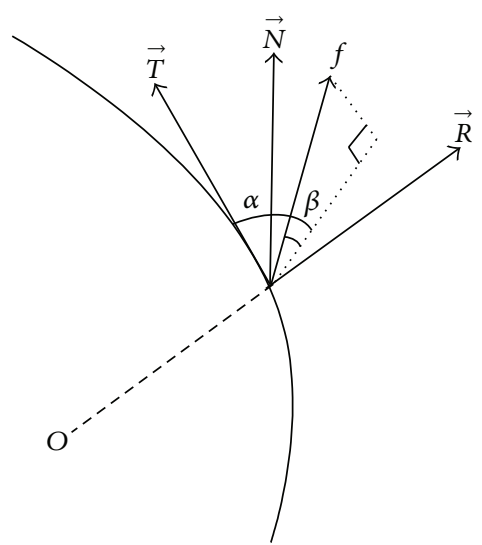

FIgURE 2: Coordinate frame.

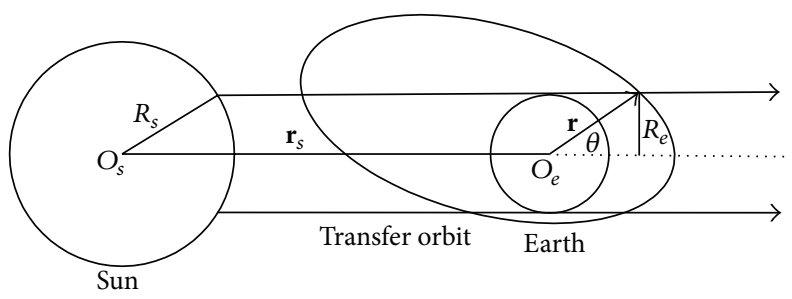

FIGURE 3: Cylindrical projection model.

$$
\begin{aligned}
& \dot{h}_{x}=\sqrt{\frac{p}{\mu}} \frac{s^{2} f_{n}}{2 \omega} \cos L \\
& \dot{h}_{y}=\sqrt{\frac{p}{\mu} \frac{s^{2} f_{n}}{2 \omega} \sin L} \\
& \dot{L}=\sqrt{\mu p}\left(\frac{\omega}{p}\right)^{2}+\frac{1}{\omega} \sqrt{\frac{p}{\mu}}\left(h_{x} \sin L-h_{y} \cos L\right) f_{n}
\end{aligned}
$$

with $\omega=1+e_{x} \cos L+e_{y} \sin L, s^{2}=1+h_{x}^{2}+h_{y}^{2}$. The acceleration components $f_{r}, f_{t}$, and $f_{n}$ are denoted in the RTN frame (with $f_{r}$ being the normal direction, $f_{t}$ being the tangential direction, and $f_{n}$ being the direction orthogonal to the orbit plane), and $f$ is the thrust acceleration. The constraint on the control is $f \leq f_{\max }=T_{\max } / \mathrm{m}$.

To take into account the true acceleration of the thrust, we must consider the mass flow. Its evolution is given by

$$
\frac{d m}{d t}=-\frac{T}{I_{\mathrm{sp}} g_{0}},
$$

where $T$ is the thrust modulus, $I_{\text {sp }}$ the specific impulse of thruster, and $g_{0}$ the gravitational acceleration at sea-level and $g_{0}=9.80665 \mathrm{~N} / \mathrm{s}$.

As shown in Figure 2, thrust acceleration components can also be defined by acceleration $f$ and two control angles (pitch-steering angle $\alpha$ and yaw-steering angle $\beta$ ), which describe the direction of the thrust vector in relation to the velocity vector and the orbital plane, respectively. The acceleration components are expressed by

$$
\begin{aligned}
& f_{r}=f \cos \beta \sin \alpha \\
& f_{t}=f \cos \beta \cos \alpha \\
& f_{n}=f \sin \beta
\end{aligned}
$$

with $-\pi \leq \alpha \leq \pi$ and $-\pi / 2 \leq \beta \leq \pi / 2$.

2.2. Eclipse Effects. To model the electric propulsion system accurately, it is necessary to model the satellite's trajectory as it passes through the shadow of Earth. In particular, the satellite is at discharge, which leads to high power consumption when in the shadow. In order to ensure the safety of spacecraft, therefore, the thrust is 0 when the vehicle is in the shadow of Earth. A simplified Earth-shadow model, namely, the cylindrical projection model shown in Figure 3, is used to estimate the location of the shadow [17-20].

Referring to the geometry illustrated in Figure $3, \mathbf{r}_{s}$ is defined as the vector from Earth to the Sun, with norm $\left\|\mathbf{r}_{s}\right\|$, and $\mathbf{r}$ as the vector from Earth to the satellite, with norm $\|\mathbf{r}\|$. $R_{e}$ is the radius of Earth, and $R_{s}$ is the radius of the Sun.

The cone angle $\theta$ is defined by

$$
\cos \theta=\angle \mathbf{r}_{s} \mathbf{r}=\frac{\mathbf{r} \cdot \mathbf{r}_{s}}{\left(\|\mathbf{r}\|\left\|\mathbf{r}_{s}\right\|\right)}
$$




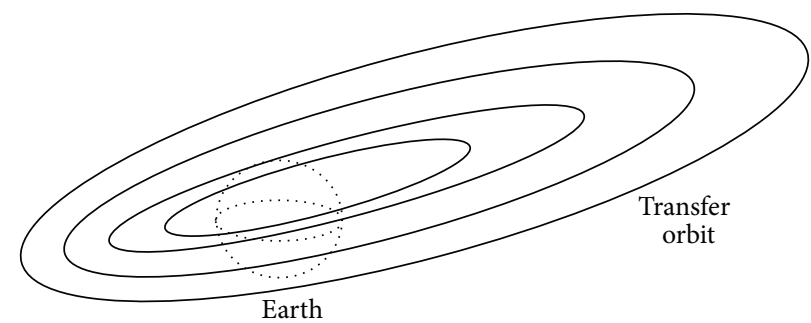

FIGURE 4: Low-thrust transfer with no radar detection.

The projected spacecraft position $\mathbf{r}$ is given by

$$
\mathbf{r}=\|\mathbf{r}\|\left[\begin{array}{c}
\cos v \cos \Omega \cos \omega-\cos v \sin \Omega \sin \omega \cos i-\sin v \cos \Omega \cos \omega-\sin v \sin \Omega \sin \omega \cos i \\
\cos v \sin \Omega \cos \omega+\cos v \cos \Omega \sin \omega \cos i-\sin v \sin \Omega \cos \omega+\sin v \cos \Omega \sin \omega \cos i \\
\cos v \sin \omega \sin i+\sin v \cos \omega \sin i
\end{array}\right] .
$$

The shadow entry and exit locations are judged by locating the cone terminators at the projected spacecraft location. The shadow can only be found when the angle satisfies

$$
\begin{aligned}
& \cos \theta \leq 0 \\
& \sin \theta<\frac{R_{e}}{r} .
\end{aligned}
$$

2.3. Perturbation. Earth's oblateness, atmospheric drag, light pressure, secondary body, and other factors in space can also perturb a satellite's motion. Among these variables, Earth's oblateness is of vital importance for predicting the trajectory of the satellite accurately. The oblate Earth perturbation is caused by the reality of Earth's shape not being perfectly spherical. The impact of Earth's oblateness due to $J_{2}$ is always taken into account in the engineering calculations [20, 21].

In terms of orbital elements, the dynamical system is

$$
\begin{aligned}
\frac{d a}{d t} & =0 \\
\frac{d e}{d t} & =0 \\
\frac{d i}{d t} & =0 \\
\frac{d \Omega}{d t} & =-\frac{3 n J_{2} R_{e}^{2}}{2\left(1-e^{2}\right) a^{2}} \cos i \\
\frac{d \omega}{d t} & =\frac{3 n J_{2} R_{e}^{2}}{4\left(1-e^{2}\right) a^{2}}\left(5 \cos ^{2} i-1\right) \\
\frac{d M}{d t} & =n-\frac{3 n J_{2} R_{e}^{2}}{4\left(1-e^{2}\right)^{3 / 2} a^{2}}\left(1-3 \cos ^{2} i\right) .
\end{aligned}
$$

The effect of Earth's oblateness (due to $J_{2}$ ) on the orbital transfer can be included by appending the perturbation to the respective right-hand sides of (5):

$$
\begin{aligned}
& f_{r}=\frac{3}{2} J_{2} \frac{u}{r^{4}} R_{E}^{2}\left(3 \sin ^{2} i \sin ^{2} u-1\right) \\
& f_{t}=-\frac{3}{2} J_{2} \frac{u}{r^{4}} R_{E}^{2} \sin ^{2} i \sin 2 u \\
& f_{n}=-J_{2} \frac{u}{r^{4}} R_{E}^{2} \sin 2 i \sin u .
\end{aligned}
$$

\section{Satellite Low Observability}

The research activity presented here is focused on the optimum algorithm and does not take into account space environment analysis, especially the low-observable constraint. The low-observable satellite is designed to minimize its frontal RCS, requiring low-observable shape design and flight attitude adjustment [6-8]. The satellite keeps its front toward Earth when it is flying over ground-based radar detection areas.

3.1. Radar Detection Area. In order to avoid reflecting radar signals directly, the scanning range of the ground-based radar should be modeled first. Figure 4 shows low-thrust GEO satellite transfer with no radar detection. Figure 5 is an illustration of that GEO satellite being detected in low-thrust transfer by a ground-based radar, and the shadow is the radar detection area. 


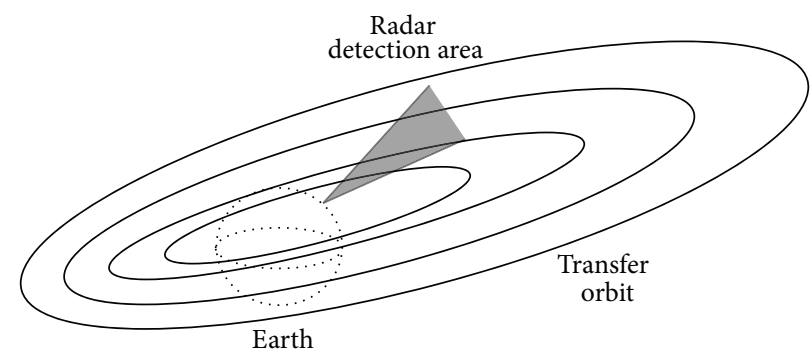

FIGURE 5: Low-thrust transfer with radar detection.

Due to the limited energy and probability of intercept, radar repeats its search for the target in a narrow area, which is modeled as a specific coverage of yaw angle, pitch angle, and operating distance, rather than the entire airspace [22-24].

The latitude and longitude of the ground station are defined, respectively, as $\lambda_{p}$ and $\delta_{p} . S$ is the track of the subsatellite point, whose right ascension and declination are denoted as $\lambda$ and $\delta . W_{e}$ is the rotation speed of Earth.

Consider

$$
\begin{aligned}
& \lambda=\operatorname{ar} \tan (\cos i \tan u)+\Omega-W_{e} t \\
& \delta=\operatorname{ar} \sin (\sin i \sin u) .
\end{aligned}
$$

The radar pitch angle $\psi_{h}$ and radar yaw angle $\phi_{h}$ are obtained by the spherical triangle. Respectively,

$$
\begin{aligned}
& \tan \psi_{h}=\frac{r \cos \gamma-R_{e}}{r \cos \gamma} \\
& \sin \phi_{h}=\frac{\sin \left(\lambda-\lambda_{p}\right) \sin \left(90^{\circ}-\delta\right)}{\sin \gamma},
\end{aligned}
$$

where $r=\left(a\left(1-e^{2}\right) /(1+e \cos v)\right) \cdot \gamma$ is the geocentric angle between the subsatellite point and observation points. It satisfies

$$
\cos \gamma=\sin \delta_{p} \sin \delta+\cos \delta_{p} \cos \delta \cos \left(\lambda-\lambda_{p}\right) .
$$

However their distance is

$$
R_{\mathrm{OP}}=\sqrt{\left(R_{e}+h\right)^{2}+R_{e}^{2}-2 R_{e}\left(R_{e}+h\right) \cos \gamma} .
$$

Suppose the radar yaw angle $\left[\sigma_{1}, \sigma_{2}\right]$, pitch angle $\left[\tau_{1}, \tau_{2}\right]$, and the operating distance $r_{\mathrm{op}}$ :

$$
\begin{aligned}
\psi_{h} & \in\left[\sigma_{1}, \sigma_{2}\right] \\
\phi_{h} & \in\left[\tau_{1}, \tau_{2}\right] \\
R_{\mathrm{OP}} & \leq r_{\mathrm{op}} .
\end{aligned}
$$

Only a satellite meeting these conditions has access to the radar detection area.

3.2. Thrust Control Angels and Satellite Attitude. Four thrusters are installed on the floor of the satellite, as shown in

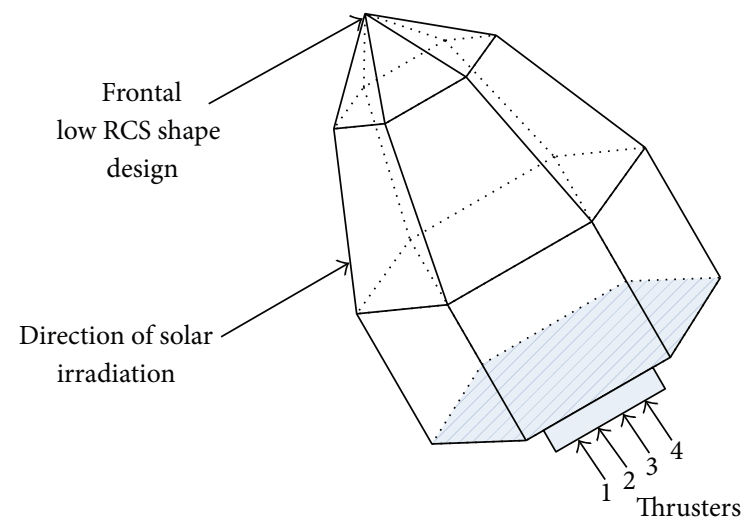

FIGURE 6: Thruster installation.

Figure 6. Two work during orbit transfer, and the other two act as a backup system. Thrust is perpendicular to the bottom of the satellite in low-thrust transfer. The satellite has a frontal RCS shape design. Assuming the maximum thrust is $F$, then thrust components in the satellite coordinates are

$$
\left[\begin{array}{lll}
F_{x} & F_{y} & F_{z}
\end{array}\right]^{T}=\left[\begin{array}{lll}
0 & F & 0
\end{array}\right]^{T} .
$$

The RTN frame is where the attitude of three axis-stabilized satellites is defined, in which thrust components are described as

$$
\begin{aligned}
& {\left[\begin{array}{lll}
F_{x_{o}} & F_{y_{o}} & F_{z_{o}}
\end{array}\right]^{T}} \\
& \quad=\left[\begin{array}{lll}
F \cos \beta \cos \alpha & F \cos \beta \sin \alpha & F \sin \beta
\end{array}\right]^{T} .
\end{aligned}
$$

Satellite attitude is related to the order of three rotations [20]. The yaw angle, roll angle, and pitch angle of the satellite are recorded as $\phi, \psi$, and $\theta$ and are derived in the order of 3-1-2. Define attitude matrix $A_{312}=R_{2}(\theta) R_{1}(\psi) R_{3}(\phi)$ and its transpose matrix $A_{312}^{-1}=R_{3}^{-1}(\phi) R_{1}^{-1}(\psi) R_{2}^{-1}(\theta)$. The relationship between satellite attitude and thrust control angles is 


$$
\begin{aligned}
{\left[\begin{array}{c}
\cos \beta \cos \alpha \\
\sin \beta \\
\cos \beta \sin \alpha
\end{array}\right] } & A_{312}^{-1}\left[\begin{array}{l}
0 \\
1 \\
0
\end{array}\right]=R_{3}^{-1}(\phi) R_{1}^{-1}(\psi) R_{2}^{-1}(\theta)\left[\begin{array}{l}
0 \\
1 \\
0
\end{array}\right] \\
& =\left[\begin{array}{ccc}
\cos \phi & -\sin \phi & 0 \\
\sin \phi & \cos \phi & 0 \\
0 & 0 & 1
\end{array}\right]\left[\begin{array}{ccc}
1 & 0 & 0 \\
0 & \cos \psi & -\sin \psi \\
0 & \sin \psi & \cos \psi
\end{array}\right]\left[\begin{array}{ccc}
\cos \theta & 0 & \sin \theta \\
0 & 1 & 0 \\
-\sin \theta & 0 & \cos \theta
\end{array}\right]\left[\begin{array}{l}
0 \\
1 \\
0
\end{array}\right] \\
& {\left[\begin{array}{ccc}
\cos \phi \cos \theta-\sin \phi \sin \psi \sin \theta & -\sin \phi \cos \psi & \cos \phi \sin \theta+\sin \phi \sin \psi \cos \theta \\
\sin \phi \cos \theta+\cos \phi \sin \psi \sin \theta & \cos \phi \cos \psi & \sin \phi \sin \theta-\cos \phi \sin \psi \cos \theta \\
-\sin \phi \sin \theta & \sin \psi & \cos \psi \cos \theta
\end{array}\right]\left[\begin{array}{l}
0 \\
1 \\
0
\end{array}\right]=\left[\begin{array}{c}
-\sin \phi \cos \psi \\
\cos \phi \cos \psi \\
\sin \psi
\end{array}\right] }
\end{aligned}
$$

It is ultimately expressed as

$$
\begin{aligned}
\cos \beta \cos \alpha & =-\sin \phi \cos \psi \\
\sin \beta & =\cos \phi \cos \psi \\
\cos \beta \sin \alpha & =\sin \psi .
\end{aligned}
$$

The attitude adjustment range in the radar irradiation area is assumed to be

$$
\begin{aligned}
& \phi_{\min }<\phi<\phi_{\max } \\
& \psi_{\min }<\psi<\psi_{\max } \\
& \theta_{\min }<\theta<\theta_{\max } .
\end{aligned}
$$

The corresponding acceleration thrust components can be obtained by

$$
\begin{aligned}
& f_{r}=f \cos \beta \sin \alpha=-f \sin \phi \cos \psi \\
& f_{t}=f \cos \beta \cos \alpha=f \cos \phi \cos \psi \\
& f_{n}=f \sin \beta=f \sin \psi .
\end{aligned}
$$

To realize low RCS toward Earth, our paper sets the optimal satellite attitude at a range of $\pm 5^{\circ}$, which can be adjusted according to the real simulation and test. Consider

$$
\begin{aligned}
& \phi \in\left[-5^{\circ}, 5^{\circ}\right] \\
& \psi \in\left[-5^{\circ}, 5^{\circ}\right] \\
& \theta \in\left[175^{\circ}, 185^{\circ}\right] .
\end{aligned}
$$

\section{Low-Thrust GEO Transfer Design}

The goal of this paper is to design a minimum-time transfer for geostationary spacecraft equipped with electric propulsion systems. The transfer problem is thus to find an essentially bound control to reduce eccentricity and inclination and raise the semimajor axis.
After simplifying (1), they fall into

$$
\begin{aligned}
\dot{a}= & \frac{2 a^{2}}{h}\left[e \sin v f_{r}+(1+e \cos v) f_{t}\right] \\
\dot{e}= & \frac{p}{h}\left[\sin v f_{r}+\left(\cos v+\frac{e+\cos v}{1+e \cos v}\right) f_{t}\right] \\
\dot{i}= & \frac{r}{h} \cos (\omega+v) f_{n} \\
\dot{\Omega}= & \frac{r \sin (\omega+v)}{h \sin i} f_{n} \\
\dot{\omega}= & -\frac{p \cos v}{h e} f_{r}+\frac{(p+r) \sin v}{h e} f_{t} \\
& +\frac{r \sin v(\omega+v) \cos i}{h \sin i} f_{n}
\end{aligned}
$$

$$
\dot{M}=n+\frac{1}{a e h}\left[(p \cos v-2 e r) f_{r}-(p+r) \sin v f_{t}\right] \text {. }
$$

The acceleration component $f_{n}$ only contributes to a decrease of inclination. The change of the semimajor axis and eccentricity are both related to $f_{r}$ and $f_{t} . f_{r}$ and $f_{t}$ have a greater effect on the semimajor axis than eccentricity.

First, a parameter $k$ is introduced to isolate $f_{n}$ from $f$ :

$$
\begin{aligned}
& f_{r t}=\sqrt{f_{r}^{2}+f_{t}^{2}}=f \cdot k \\
& f_{n}=f \sqrt{1-k^{2}}
\end{aligned}
$$

with $0 \leq k \leq 1$.

Then, a control law that reduces eccentricity quickly is derived by observing the time-rate equation for the semimajor axis and eccentricity. Define $e_{p 1}=\sin v$ and $e_{p 2}=$ $\cos v+(e+\cos v) /(1+e \cos v)$. The law is given by

$$
\begin{aligned}
& \left.\begin{array}{c}
f_{r}=f \cdot k \\
f_{t}=0
\end{array}\right\}\left|e_{p 1}\right|>\left|e_{p 2}\right| \\
& \left.\begin{array}{c}
f_{r}=0 \\
f_{t}=f \cdot k
\end{array}\right\}\left|e_{p 1}\right| \leq\left|e_{p 2}\right| .
\end{aligned}
$$


TABLE 1: Thrust modes in low-thrust transfer.

\begin{tabular}{|c|c|c|c|c|c|}
\hline Direction & \multicolumn{5}{|c|}{ Modulus } \\
\hline \multirow{2}{*}{ Condition } & Mode 1 & Mode 2 & Mode 3 & Mode 4 & Mode 5 \\
\hline & $k \neq 1$ & $k=1$ & $k=1$ & Earth shadow & Low observability \\
\hline \multicolumn{6}{|c|}{$i_{p}=\cos (\omega+v)$} \\
\hline $\begin{array}{l}i_{p}>0 \\
i_{p} \leq 0\end{array}$ & $\begin{array}{l}f_{n}=-f \sqrt{1-k^{2}} \\
f_{n}=f \sqrt{1-k^{2}}\end{array}$ & $f_{n}=0$ & $f_{n}=0$ & & $f_{n}=0$ \\
\hline$\left|e_{p 1}\right|>\left|e_{p 2}\right|$ & & & & $f_{n}=0$ & \\
\hline$e_{p 1} \geq 0$ & $f_{r}=-f \cdot k, f_{t}=0$ & $f_{r}=-f, f_{t}=0$ & & $f_{r}=0$ & $f_{r}=0, f_{t}=0$ \\
\hline $\begin{array}{c}e_{p 1}<0 \\
\left|e_{p 1}\right| \leq\left|e_{p 2}\right|\end{array}$ & $f_{r}=f \cdot k, f_{t}=0$ & $f_{r}=f, f_{t}=0$ & $\begin{array}{l}f_{r}=f \\
f_{t}=0\end{array}$ & $f_{t}=0$ & $f_{r}=0, f_{t}=0$ \\
\hline$e_{p 2} \geq 0$ & $f_{r}=0, f_{t}=-f \cdot k$ & $f_{r}=0, f_{t}=-f$ & & & $f_{r}=0, f_{t}=0$ \\
\hline$e_{p 2}<0$ & $f_{r}=0, f_{t}=f \cdot k$ & $f_{r}=0, f_{t}=f$ & & & $f_{r}=0, f_{t}=f$ \\
\hline
\end{tabular}

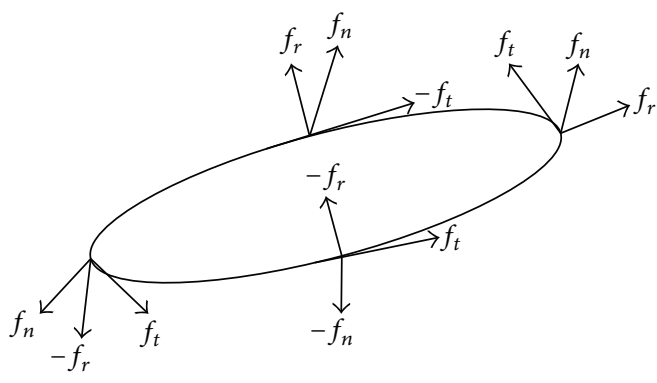

FIGURE 7: Reduction of inclination.

Five thrust modes and both their corresponding modulus and direction are summed up in Table 1.

As stated, our transfer problem is parameterized, and the control is given according to $i_{p}, e_{p 1}$, and $e_{p 2}$, which are determined by the argument of perigee and the true anomaly. Because the parameter $k$ is between 0 and 1 , it is convenient to optimize it to minimize the transfer time by a simple traversal, which is a selection of $k$ from 0 to 1 by varying it in a prefixed step. As Table 1 details fully, our method for achieving terminal orbit is performed in three steps.

Step 1 (reduction of inclination). The thrust mode relies on mode 1 to reduce inclination by rational $k$, along with the simultaneous targeting of the semimajor axis and eccentricity. The thrust form is shown in Figure 7.

Step 2 (raising the semimajor axis). Mode 2 represents a law used to control the semimajor axis and the eccentricity when the inclination reaches the target value. The thrust operates as shown in Figure 8.

Step 3 (reducing eccentricity). While the semimajor axis reaches its target, $f_{t}$ remains at 0 to ensure the stability of the semimajor axis. $f_{r}$ is used to reduce eccentricity in mode 3 , as illustrated in Figure 9.

Step 4. Mode 4 is the thrust model for Earth shadow, according to formula (9) to realize real-time judgment of shadow, in which thrust is 0 .

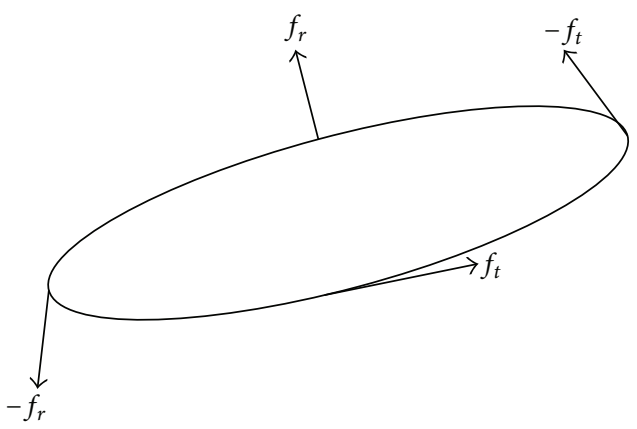

FIGURE 8: Raising semimajor axis.

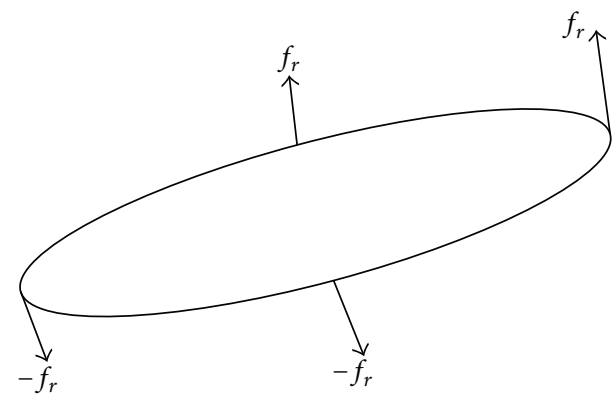

FIGURE 9: Reducing eccentricity.

Step 5. Mode 5 is used for the radar detection area to realize satellite low observability. The satellite adjusts to its best attitude, corresponding to thrust components $f_{r}=0, f_{n}=0$, and, if $e_{p 2}<0, f_{t}=f$; otherwise, $f_{t}=0$, because $f_{t}=f$ contributes to both the decrease of eccentricity and the increase of the semimajor axis when $e_{p 2}<0$ (Figure 10).

In short, the method described in this paper determines a control depending on a parameter and the state of current orbit. Our control law is simple, with only one uncertain control parameter, whose optimization is so convenient that our minimum-time transfer problem is greatly simplified. 


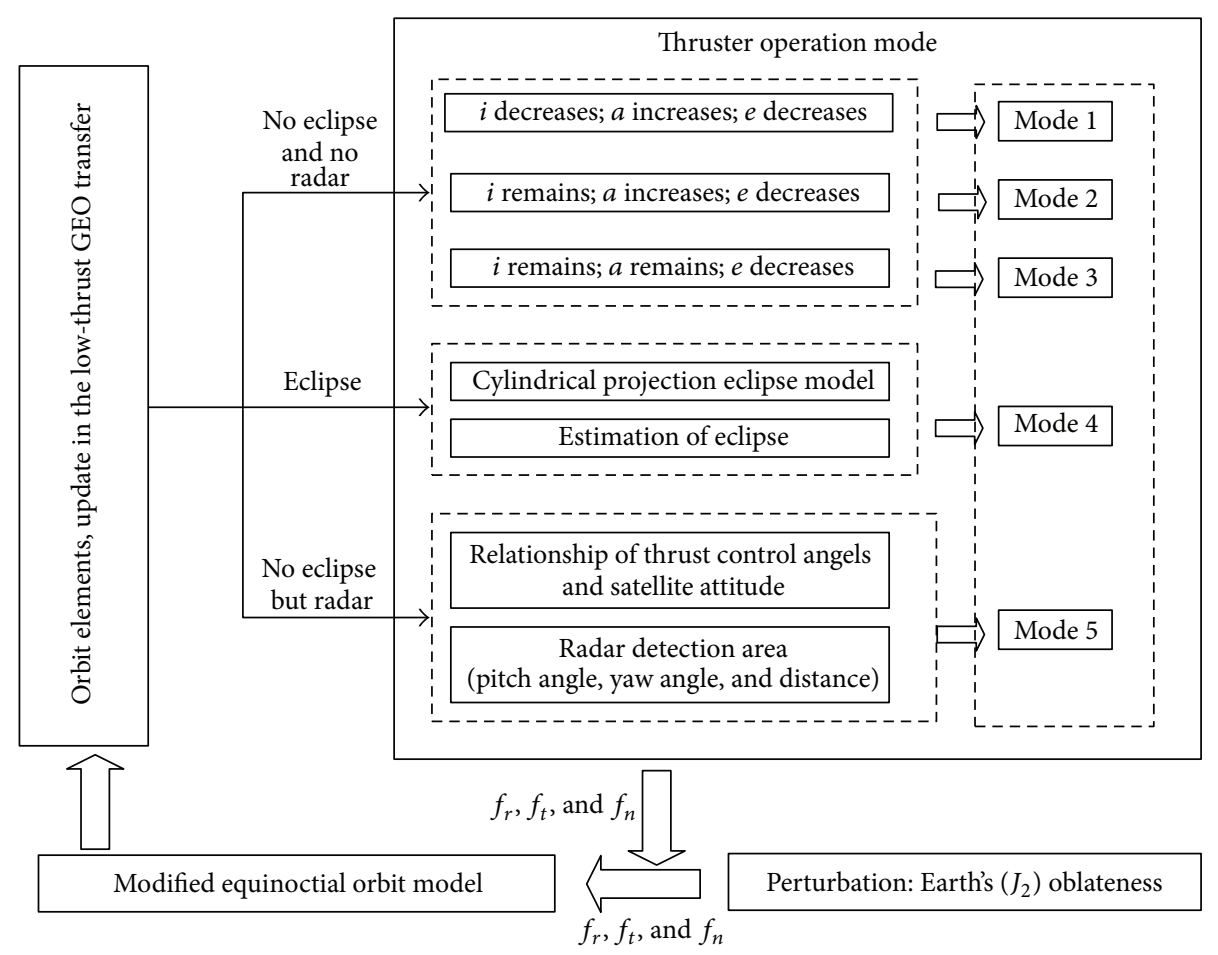

FIgURE 10: Overall scheme.

\section{Simulation}

5.1. Initial Parameter. The ideal attitude of the satellite was assumed to have the range of its yaw angle, roll angle, and pitch angle as $\left[-5^{\circ}, 5^{\circ}\right],\left[-5^{\circ}, 5^{\circ}\right]$, and $\left[175^{\circ}, 185^{\circ}\right]$. The yaw angle of each radar ranges from $-60^{\circ}$ to $60^{\circ}$, pitch angle ranges from 0 to $90^{\circ}$, and operating distance was $15,000 \mathrm{~km}$. They were located in the following places (longitude/latitude): $\left(65^{\circ} \mathrm{E}, 35^{\circ} \mathrm{N}\right),\left(30^{\circ} \mathrm{E}, 75^{\circ} \mathrm{N}\right),\left(75^{\circ} \mathrm{E}, 75^{\circ} \mathrm{N}\right),\left(120^{\circ} \mathrm{E}, 75^{\circ} \mathrm{N}\right)$, $\left(165^{\circ} \mathrm{E}, 75^{\circ} \mathrm{N}\right),\left(15^{\circ} \mathrm{E}, 75^{\circ} \mathrm{N}\right),\left(60^{\circ} \mathrm{W}, 75^{\circ} \mathrm{N}\right),\left(105^{\circ} \mathrm{W}, 75^{\circ} \mathrm{N}\right)$, and $\left(150^{\circ} \mathrm{W}, 75^{\circ} \mathrm{N}\right) ;\left(30^{\circ} \mathrm{E}, 75^{\circ} \mathrm{S}\right),\left(75^{\circ} \mathrm{E}, 75^{\circ} \mathrm{S}\right),\left(120^{\circ} \mathrm{E}, 75^{\circ} \mathrm{S}\right),\left(165^{\circ} \mathrm{E}\right.$, $\left.75^{\circ} \mathrm{S}\right),\left(15^{\circ} \mathrm{E}, 75^{\circ} \mathrm{S}\right),\left(60^{\circ} \mathrm{W}, 75^{\circ} \mathrm{S}\right),\left(105^{\circ} \mathrm{W}, 75^{\circ} \mathrm{S}\right)$, and $\left(150^{\circ} \mathrm{W}\right.$, $\left.75^{\circ} \mathrm{S}\right)$; $\left(30^{\circ} \mathrm{E}, 45^{\circ} \mathrm{N}\right),\left(75^{\circ} \mathrm{E}, 45^{\circ} \mathrm{N}\right),\left(120^{\circ} \mathrm{E}, 45^{\circ} \mathrm{N}\right),\left(165^{\circ} \mathrm{E}\right.$, $\left.45^{\circ} \mathrm{N}\right),\left(15^{\circ} \mathrm{E}, 45^{\circ} \mathrm{N}\right),\left(60^{\circ} \mathrm{W}, 45^{\circ} \mathrm{N}\right),\left(105^{\circ} \mathrm{W}, 45^{\circ} \mathrm{N}\right)$, and $\left(150^{\circ} \mathrm{W}\right.$, $\left.45^{\circ} \mathrm{N}\right)$; $\left(30^{\circ} \mathrm{E}, 45^{\circ} \mathrm{S}\right),\left(75^{\circ} \mathrm{E}, 45^{\circ} \mathrm{N}\right),\left(120^{\circ} \mathrm{E}, 45^{\circ} \mathrm{S}\right),\left(165^{\circ} \mathrm{E}, 45^{\circ} \mathrm{S}\right)$, $\left(15^{\circ} \mathrm{E}, 45^{\circ} \mathrm{S}\right),\left(60^{\circ} \mathrm{W}, 45^{\circ} \mathrm{S}\right),\left(105^{\circ} \mathrm{W}, 45^{\circ} \mathrm{S}\right)$, and $\left(150^{\circ} \mathrm{W}, 45^{\circ} \mathrm{S}\right)$; $\left(30^{\circ} \mathrm{E}, 15^{\circ} \mathrm{N}\right),\left(75^{\circ} \mathrm{E}, 15^{\circ} \mathrm{N}\right),\left(120^{\circ} \mathrm{E}, 15^{\circ} \mathrm{N}\right),\left(165^{\circ} \mathrm{E}, 15^{\circ} \mathrm{N}\right),\left(15^{\circ} \mathrm{E}\right.$, $\left.15^{\circ} \mathrm{N}\right),\left(60^{\circ} \mathrm{W}, 15^{\circ} \mathrm{N}\right),\left(105^{\circ} \mathrm{W}, 15^{\circ} \mathrm{N}\right)$, and $\left(150^{\circ} \mathrm{W}, 15^{\circ} \mathrm{N}\right) ;\left(30^{\circ} \mathrm{E}\right.$, $\left.15^{\circ} \mathrm{S}\right),\left(75^{\circ} \mathrm{E}, 15^{\circ} \mathrm{S}\right),\left(120^{\circ} \mathrm{E}, 15^{\circ} \mathrm{S}\right),\left(165^{\circ} \mathrm{E}, 15^{\circ} \mathrm{S}\right),\left(15^{\circ} \mathrm{E}, 15^{\circ} \mathrm{S}\right)$, $\left(60^{\circ} \mathrm{W}, 15^{\circ} \mathrm{S}\right),\left(105^{\circ} \mathrm{W}, 15^{\circ} \mathrm{S}\right)$, and $\left(150^{\circ} \mathrm{W}, 15^{\circ} \mathrm{S}\right)$.

The satellite's initial mass $m_{0}=2600 \mathrm{~kg}$ was assumed to be equipped with four thrusters with a specific impulse of $I_{\mathrm{sp}}=1600 \mathrm{~s}$ and a maximum thrust of $F=80 \mathrm{mN}$. The GTO departure date was fixed as 1 June 2008 for shadow calculations. Table 2 displays the initial GTO and GEO boundary conditions.

5.2. Thrust and Control Angles. Figures 11 and 12 show the time-evolution of thrust and thrust control angles in the orbit transfer, in which Earth shadow and oblateness $\left(J_{2}\right)$ effects were considered with no low-observable constraint.
TABLE 2: Orbit parameters.

\begin{tabular}{lcc}
\hline Orbit parameters & $\begin{array}{c}\text { Initial orbit } \\
\text { GTO }\end{array}$ & $\begin{array}{c}\text { Final orbit } \\
\text { GEO }\end{array}$ \\
\hline Semimajor axis $a(\mathrm{~km})$ & 24731 & 42164 \\
Inclination $i(\mathrm{deg})$ & 28.5 & 0 \\
Eccentricity $e$ & 0.7194 & 0 \\
Right ascension $\Omega(\mathrm{deg})$ & 1.7104 & Free \\
Argument of perigee $\omega(\mathrm{deg})$ & 179.6 & Free \\
Mean anomaly $M(\mathrm{deg})$ & 2.9044 & Free \\
\hline
\end{tabular}

In contrast, Figures 13 and 14 show results taking into consideration all of the aforementioned factors.

As can be seen in Figures 11-14, the satellite flew over the radar detection area three times in these six orbit periods, respectively, $6.263 \times 10^{6} \sim 6.294 \times 10^{6} \mathrm{~s}, 6.363 \times 10^{6} \sim$ $6.377 \times 10^{6} \mathrm{~s}$, and $6.44 \times 10^{6} \sim 6.465 \times 10^{6} \mathrm{~s}$. The thrust components were $f_{r}=0, f_{t}=f$, and $f_{n}=0$. The thruster operated in mode 5 to maintain low observability of the satellite (Table 3).

5.3. Numerical Results. To demonstrate the validity of our proposed method, four scenarios are presented in Table 4. Case 1 represents a transfer based on the optimal control method 1, using Pontryagin's maximum principle to find the optimum control law [14]. Cases 2 and 3 represent transfers based on our method. Case 3 involves Earth shadow and oblateness $\left(\mathrm{J}_{2}\right)$ effects. Cases 4 and 5 involve Earth shadow, oblateness $\left(J_{2}\right)$ effects, and low observability. Case 4 considers 

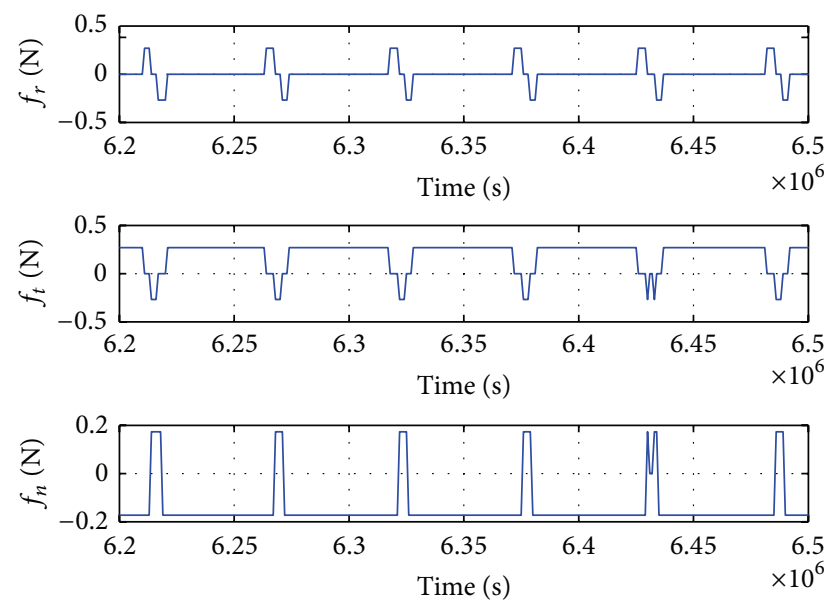

FIGURE 11: Time-evolution of thrust with no radar detection.
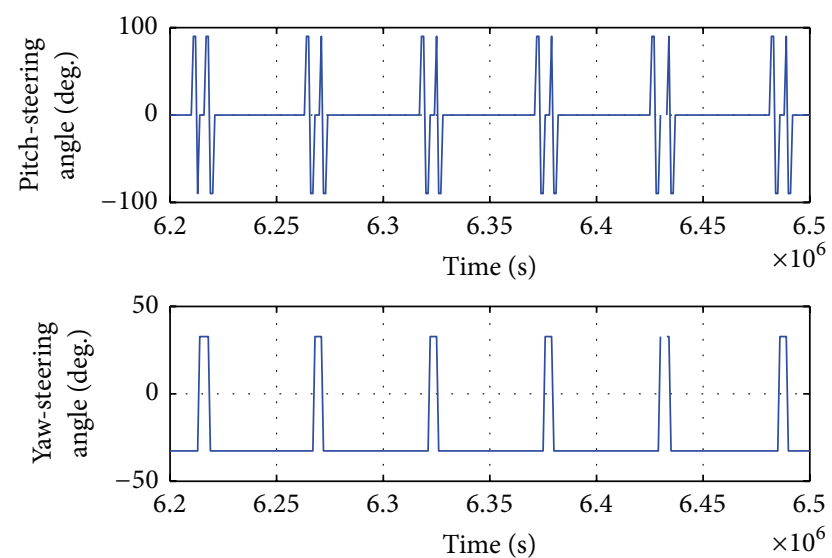

FIgURE 12: Time-evolution of thrust control angles with no radar detection.

only one radar detection area $\left(65^{\circ} \mathrm{E}, 35^{\circ} \mathrm{N}\right)$, while Case 5 includes other 48 areas.

The results of these four cases are summarized in detail in Table 4. The time-evolution of state for Case 3 is presented in Figures 15-17. The time-evolution of state for Case 4 is shown in Figures 18-20. The time-evolution of state for Case 5 is presented in Figures 21-23. GTO-GEO transfer times were 230 days and 245 days for the optimal method and our method, respectively. Case 4 led to a 288-day transfer, requiring $507 \mathrm{~kg}$ of fuel. Case 5 was a 410-day transfer consuming $723 \mathrm{~kg}$ of fuel. Compared with the results of the optimal control, our method clearly offered performance near the results of optimal method with no ground estimation, was particularly flexible when orbit elements changed, and presented no twopoint boundary problem and a low computational burden.

\section{Conclusion}

A new method based on control parameter analysis has been used to design a low-thrust orbit transfer. The optimization of the parameter introduced here is utilized to solve the optimal
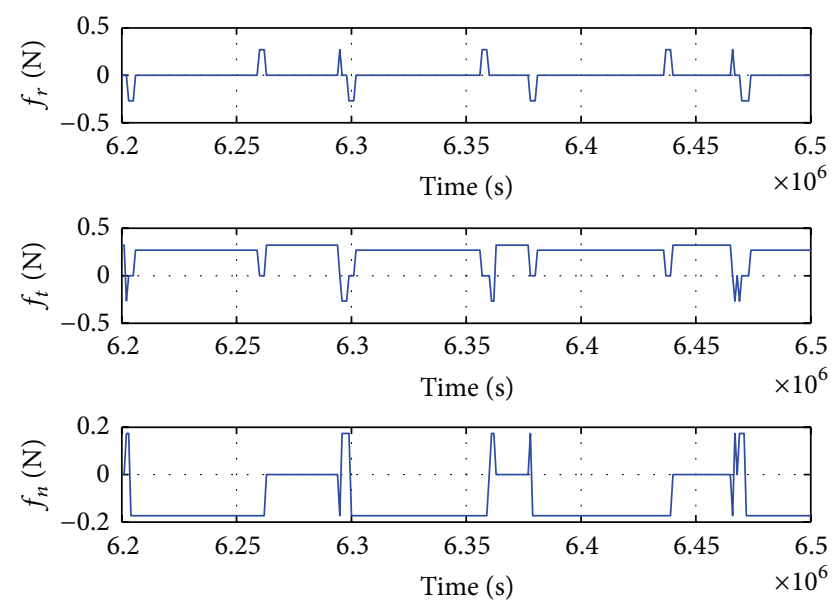

FIGURE 13: Time-evolution of thrust with radar detection.
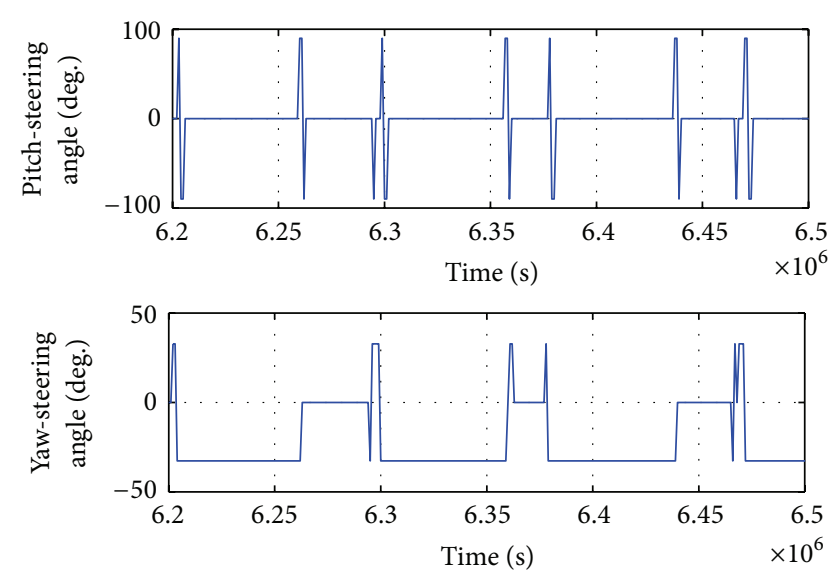

FIGURE 14: Time-evolution of thrust control angles with radar detection.

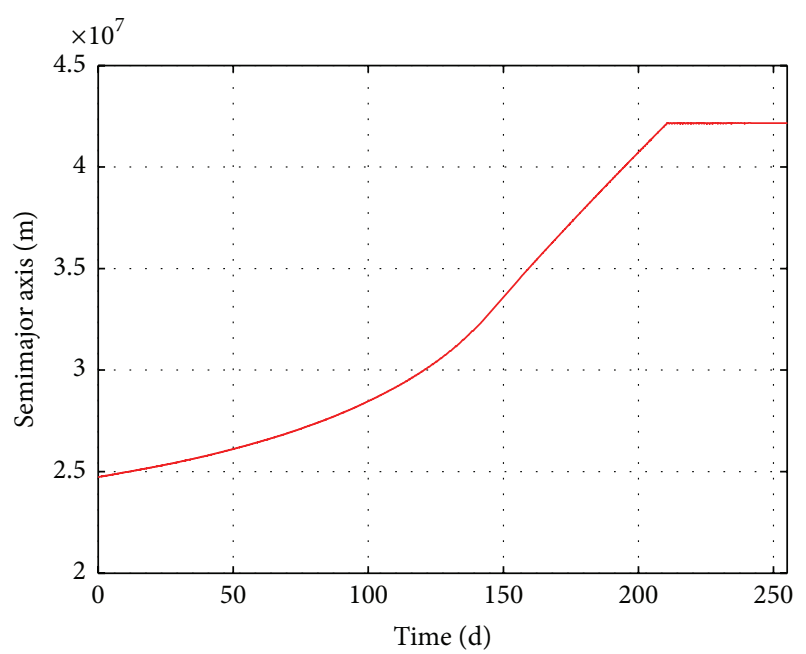

Figure 15: Time-evolution of the semimajor axis of Case 3. 
TABLE 3: Low-thrust transfer with low observability.

\begin{tabular}{lcccc}
\hline & Start/stop time $(\mathrm{s})$ & Total time $(\mathrm{s})$ & Thrust componets $\mathrm{N}$ & Control angels \\
\hline 1 & $6.263 \times 10^{6} \sim 6.294 \times 10^{6} \mathrm{~s}$ & $3.1 \times 10^{4}$ & $f_{r}=0, f_{t}=f$, and $f_{n}=0$ & $\alpha=0, \beta=0$ \\
2 & $6.363 \times 10^{6} \sim 6.377 \times 10^{6} \mathrm{~s}$ & $1.4 \times 10^{4}$ & $f_{r}=0, f_{t}=f$, and $f_{n}=0$ & $\alpha=0, \beta=0$ \\
3 & $6.44 \times 10^{6} \sim 6.465 \times 10^{6} \mathrm{~s}$ & $2.5 \times 10^{4}$ & $f_{r}=0, f_{t}=f$, and $f_{n}=0$ & $\alpha=0, \beta=0$ \\
\hline
\end{tabular}

TABLE 4: Fuel consumption and transfer days.

\begin{tabular}{lccccc}
\hline \multirow{2}{*}{ Mission } & \multicolumn{3}{c}{ GTO $\rightarrow$ GEO } & Case 4 & Case 5 \\
\hline Fuel consumption $\Delta m(\mathrm{~kg})$ & Case 1 & Case 2 & Case 3 & 443 & 723 \\
Transfer days $\Delta t(\mathrm{~d})$ & 404 & 431 & 251 & 288 & 410 \\
\hline
\end{tabular}

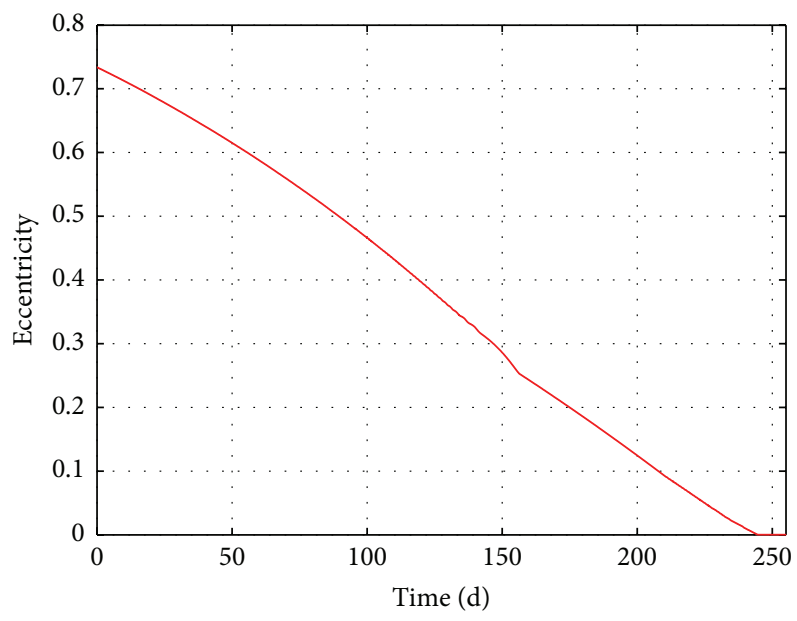

FIGURE 16: Time-evolution of the eccentricity of Case 3.

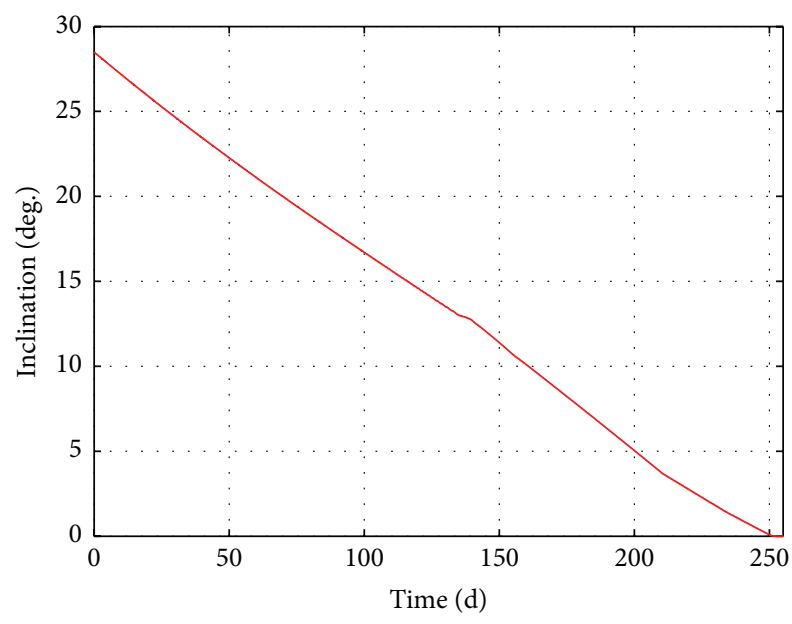

Figure 17: Time-evolution of the inclination of Case 3.

control problem. Satellite low observability was considered in GEO transfers, and the effects of Earth shadow and oblateness were also included in our method.

This approach possesses three important features: (1) The approach is characterized by simplicity and feasibility,

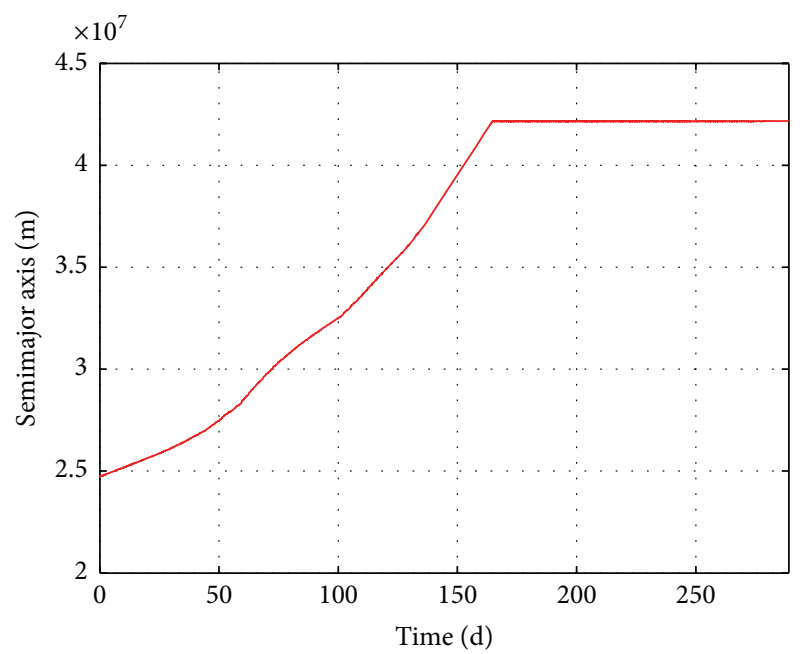

FIgURE 18: Time-evolution of the semimajor axis of Case 4.

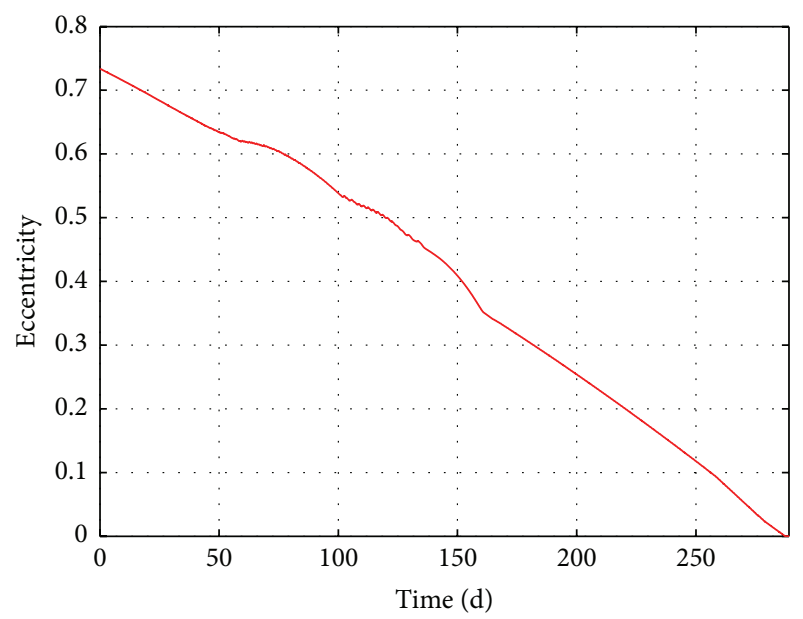

FIgURE 19: Time-evolution of the eccentricity of Case 4.

on-board real-time solution, less calculation, and no initial guess for convergence, (2) the influence of Earth shadow and perturbation is taken into account, proving its strong fault tolerance, and (3) a simple but effective approach is used to 


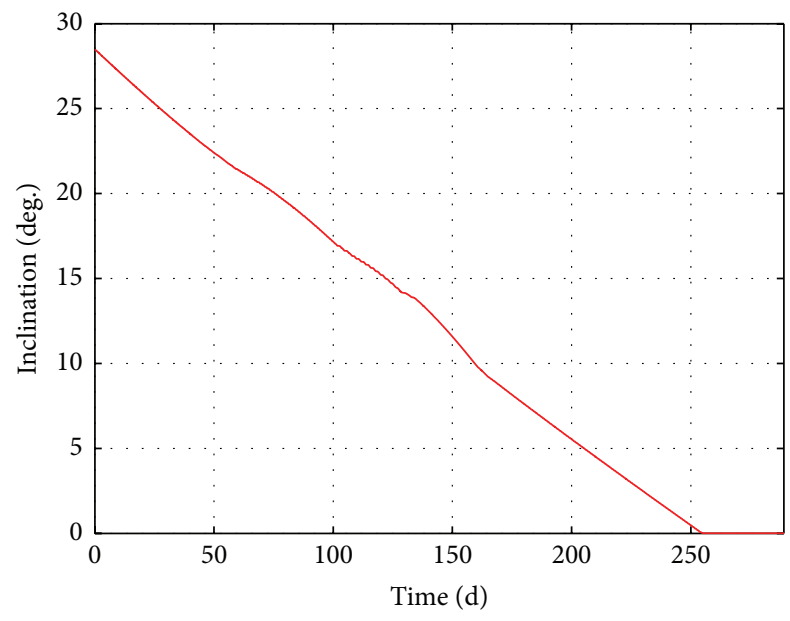

FIgURE 20: Time-evolution of the inclination of Case 4.

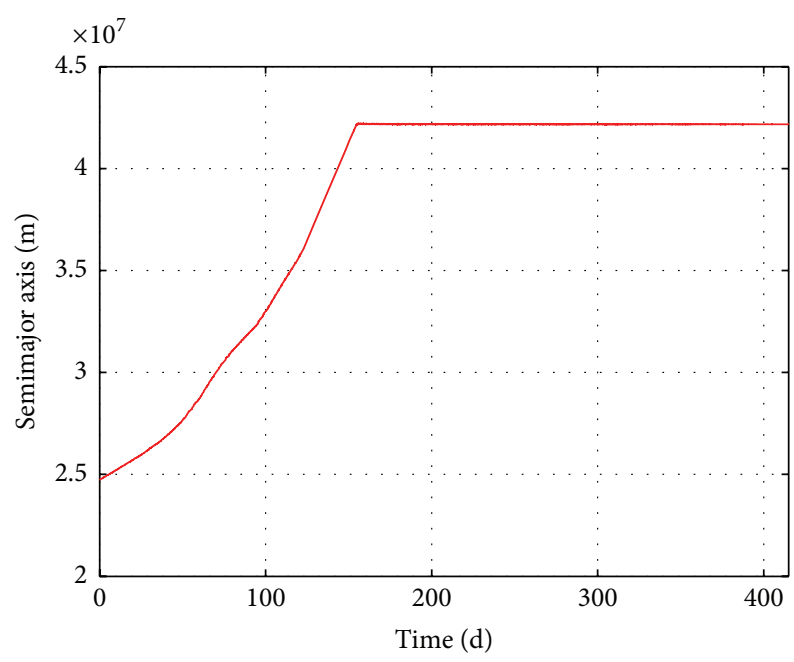

FIgURE 21: Time-evolution of the semimajor axis of Case 5.

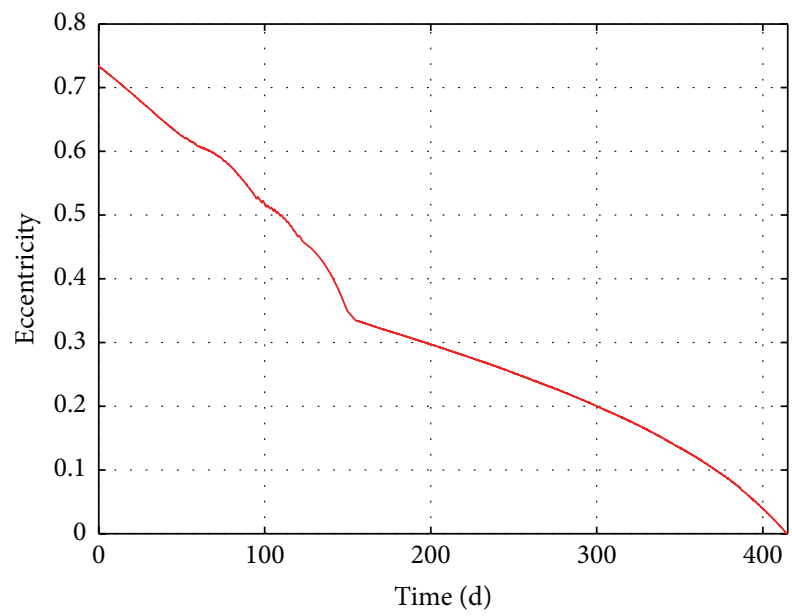

FIGURE 22: Time-evolution of the eccentricity of Case 5.

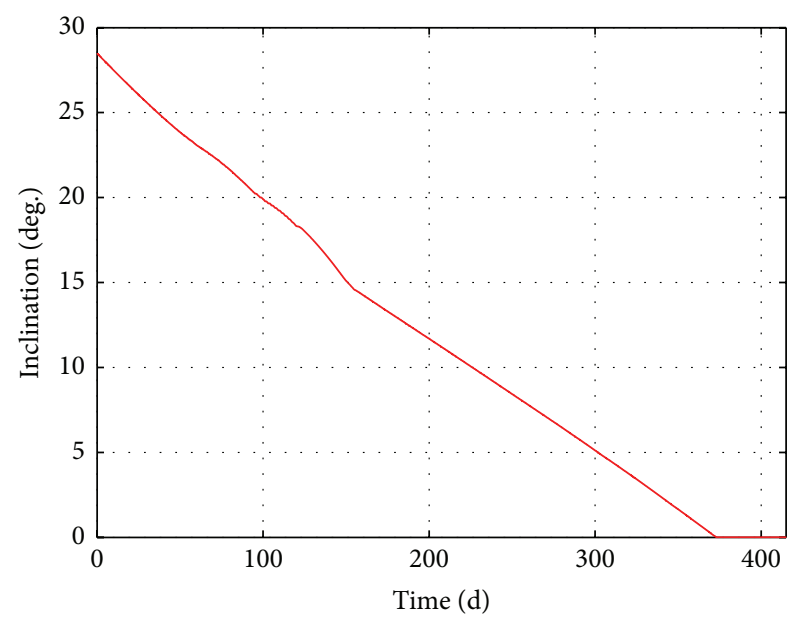

FIGURE 23: Time-evolution of the inclination of Case 5.

achieve satellite low observability and reduce the threat of radar. In general, this trajectory method would serve as a useful preliminary design for GEO mission designers.

\section{Conflict of Interests}

The authors declare that there is no conflict of interests regarding the publication of this paper.

\section{Acknowledgments}

The paper is supported by the open funding project of State Key Laboratory of Virtual Reality Technology and Systems, Beihang University (Grant no. BUAA-VR-14KF-06) and National Natural Science Foundation of China (no. 61203188).

\section{References}

[1] C. Wang and W. Liao, "Development status and application in space attack-defense of stealthy satellite," Aerospace Electronic Warfare, vol. 27, no. 4, pp. 17-19, 2011.

[2] K. Su and J. Zhou, "Development of satellite stealth technology," Stealth Technology, no. 3, pp. 9-14, 2008.

[3] J. Zhang, Y. Gen, X. Qin, and Y. Zhao, "Analysis of detection efficiency of space target surveillance radar to low-orbit satellite," Radar Science and Technology, vol. 7, no. 1, pp. 10-14, 2009.

[4] L. Meng, X. Zhang, and Y. Hou, "Development analysis on low detectionable characteristics of satellite," Aerospace Electronic Warfare, vol. 26, no. 1, pp. 33-36, 2010.

[5] C. Wang, M. Shane, and T. Nguyen, "Radio frequency interference from ground radars to a geostationary satellite," in Proceedings of the 20th AIAA International Communication Satellite Systems Conference and Exhibit, AIAA 2002-1948, Montreal, Canada, May 2002.

[6] K. Su, Key technology research on micro-satellite low observability [Ph.D. thesis], Nanjing University of Aeronautics and Astronautics, Nanjing, China, 2011.

[7] D. Micheli, R. Pastore, G. Giannini, A. Vricella, and M. Marchetti, "Low-cost low-observable satellites made of carbon 
nanostructured multilayers," in Proceedings of the 53rd Israel Annual Conferenceon Aerospace Sciences, Tel Aviv, Israel, March 2013.

[8] K. Su and J. J. Zhou, "Flight attitude planning for low observable micro-satellite shields," Aeronautic et Astronautic Since, vol. 32, no. 4, pp. 720-728, 2011.

[9] X. Bian and Y. Wei, "Key techniques for the application of the electric propulsion system in the geostationary satellite platform," Aerospace Control and Application, vol. 34, no. 1, pp. 20-24, 2008.

[10] P. Cui, D. Qiao, and H. Cui, Trajectory Design and Optimization of Deep Space Exploration, Science Press, 2013.

[11] G. Tang, H. Zhang, and G. Wang, Dynamics and Control of LowThrust Orbital Maneuver, Science Press, 2013.

[12] B. A. Conway, Spacecraft Trajectory Optimization, Cambridge University Press, Cambridge, UK, 2010.

[13] J. T. Betts, "Survey of numerical methods for trajectory optimization," Journal of Guidance, Control, and Dynamics, vol. 21, no. 2, pp. 193-207, 1998.

[14] J. Fourcade, S. Geffroy, and R. Epenoy, "An averaging optimal control tool for low-thrust minimum-time transfers," in Low Thrust Trajectory Optimization, CNES, 2000.

[15] G. T. Huntington, Advancement and analysis of a Gauss pseudospectral transcription for optimal control problems [Ph.D. thesis], University of Florida, Gainesville, Fla, USA, 2007.

[16] K. Yu and M. Xu, "Design for orbit-transfer based on ion propulsion," Aerospace Control and Application, vol. 39, no. 5, pp. 39-42, 2013.

[17] C. A. Kluever, "Using edelbaum's method to compute LowThrust transfers with earth-shadow eclipses," Journal of Guidance, Control, and Dynamics, vol. 34, no. 1, pp. 300-303, 2011.

[18] J. T. Betts, "Optimal low-thrust orbit transfers with eclipsing," Optimal Control Applications \& Methods, vol. 36, no. 2, pp. 218240, 2015.

[19] J. Ding, F. Qiu, and Z. Wei, "Calculating and analysis of satellite umbra based on Cartesian coordinate," Science of Surveying and Mapping, no. 1, pp. 39-40, 2010.

[20] X. Xi, W. Wang, and Y. Gao, Fundamentals of Near-Earth Spacecraft Obit, National University of Defense Technology Press, 2003.

[21] J. Yang, Q. Fan, W. Yang, and S. Chen, Dynamics and Control of Spacecraft, Aerospace Press, 1999.

[22] K. Hu and J. Luo, "Principle analysis of ground based surveillance radar to space target," Space Electronic Technology, vol. 6, no. 4, pp. 66-70, 2009.

[23] J. Zhang and G. Yang, "Analysis of detection efficiency of space target surveillance radar to low-orbit satellite," Radar Science and Technology, vol. 7, no. 1, pp. 10-13, 2009.

[24] R. Zhang, Attitude Dynamics and Control of Satellite Orbit, Beihang University Press, 1998. 

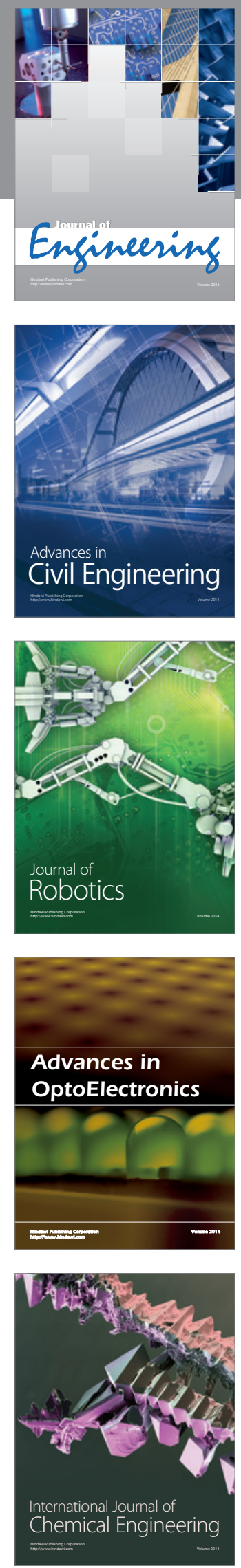

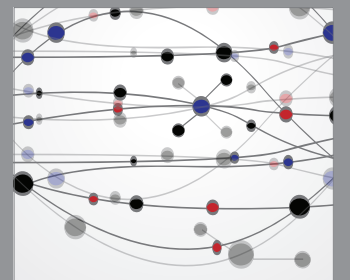

The Scientific World Journal
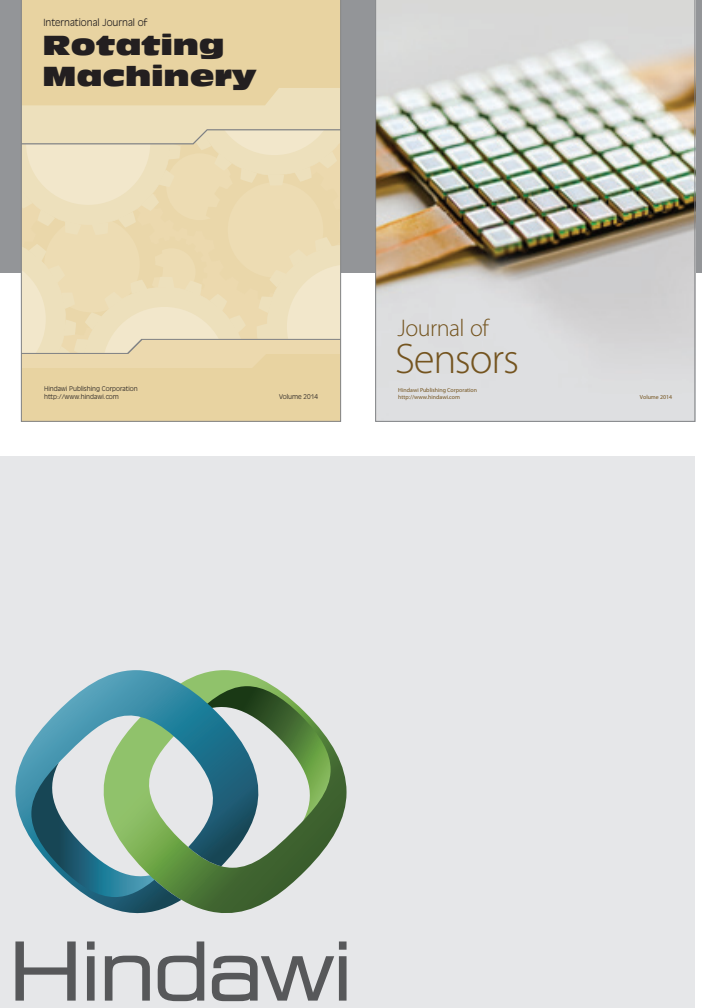

Submit your manuscripts at http://www.hindawi.com
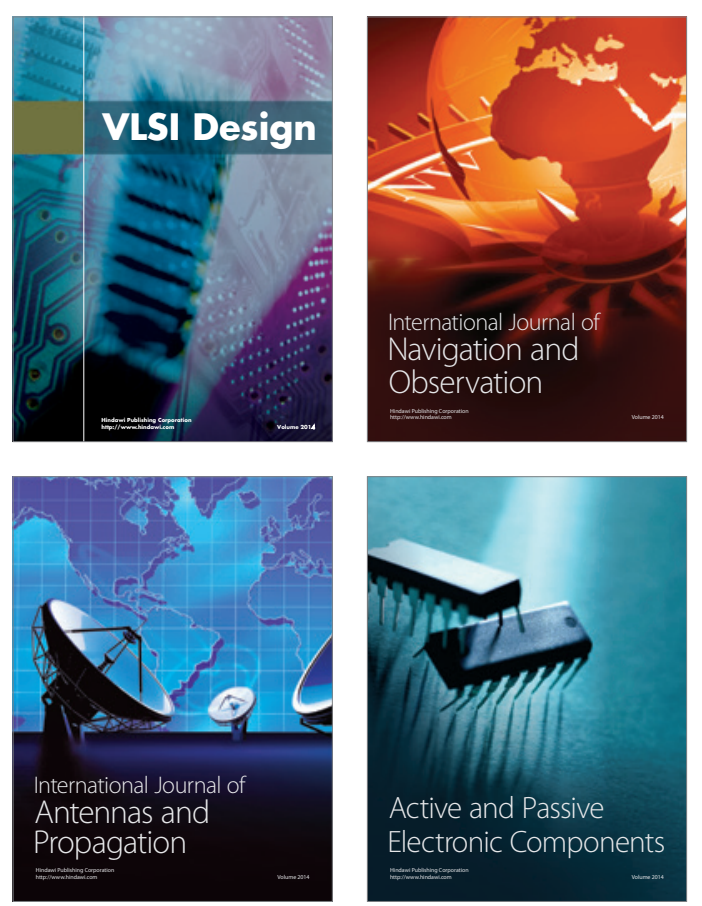
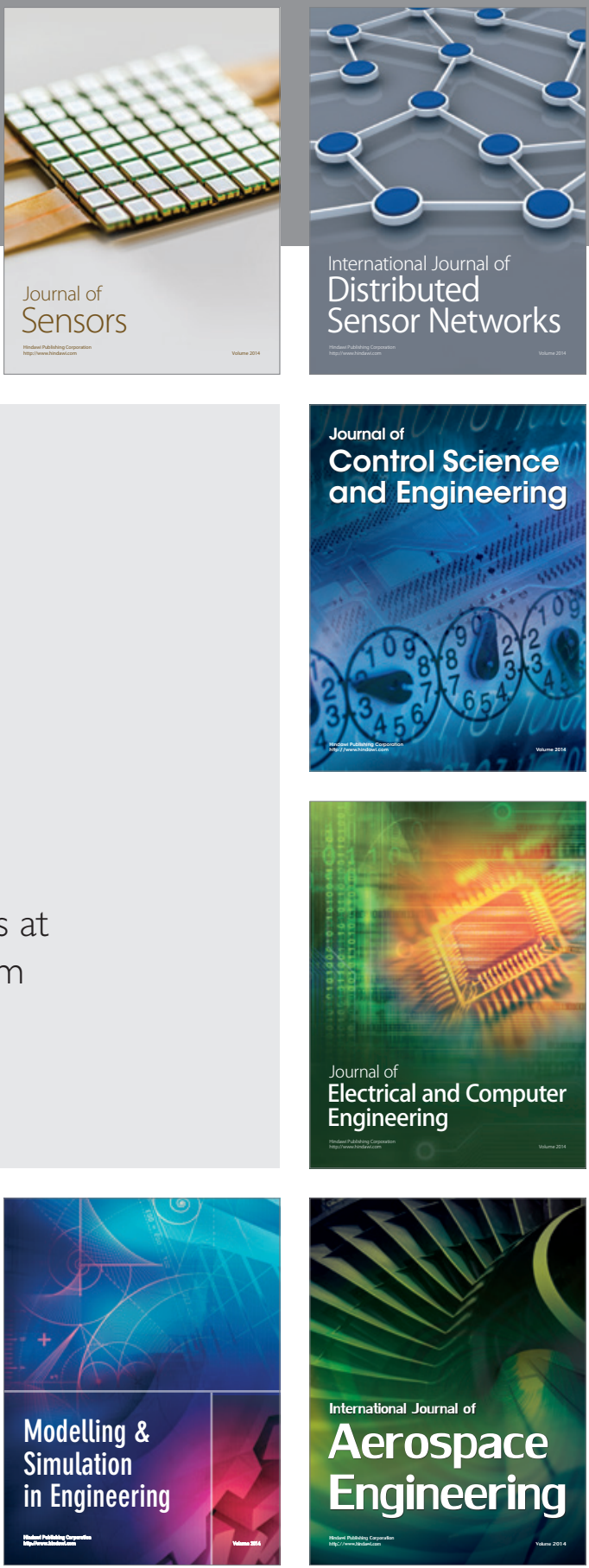

Journal of

Control Science

and Engineering
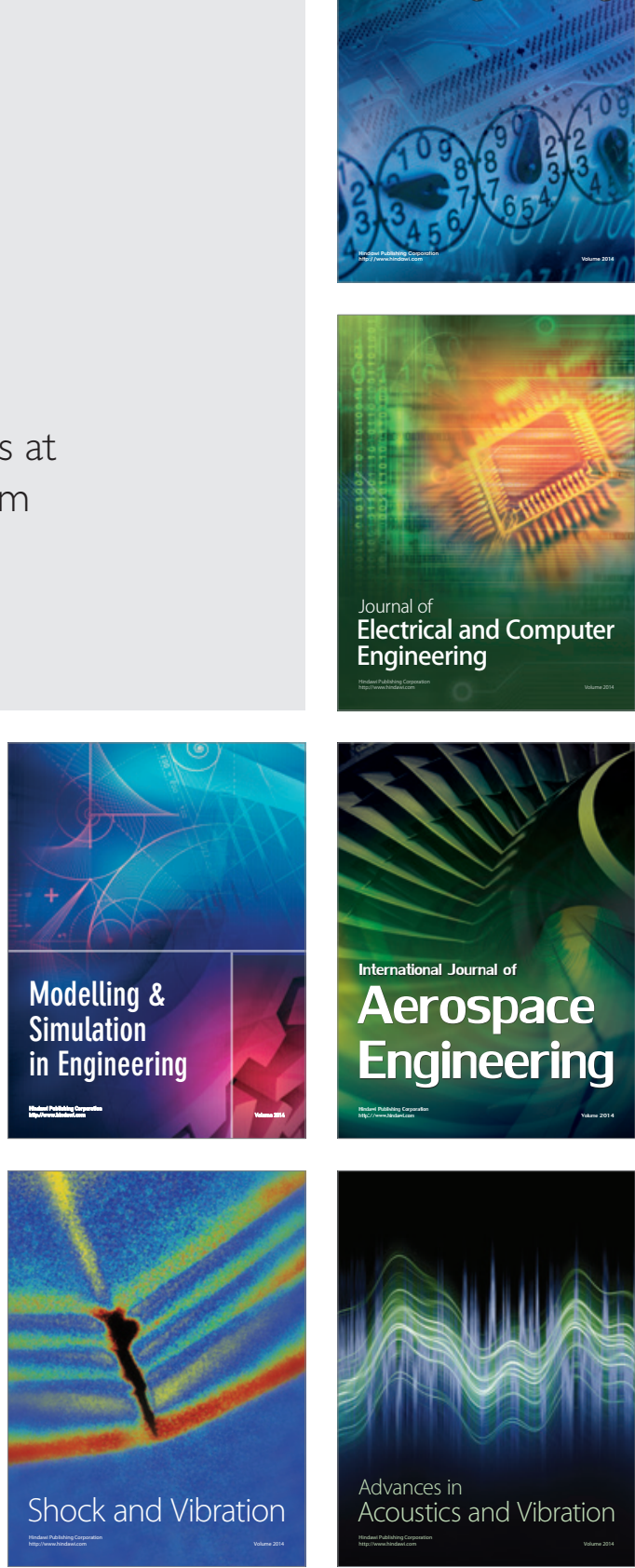